\title{
Utilização do Método da Unidade de Esforço de Produção (UEP): estudo em uma empresa de cosméticos.
}

Guilherme Luiz

Mestrado em andamento em Contabilidade pela Universidade Federal de Santa

Catarina - UFSC

Contador na Universidade Federal de Santa Catarina - UFSC Rua Valdemiro João Espíndola, 688. Aririu da Formiga. Palhoça/SC. CEP: 88134-

E-mail: guilhermeluiz_@hotmail.com

Valdirene Gasparetto

Doutorado em Engenharia de Produção pela Universidade Federal de Santa

Catarina - UFSC

Professora Adjunta da Universidade Federal de Santa Catarina - UFSC Reitor João David Ferreira Lima. Bairro Trindade. Florianópolis/SC. CEP 88040-900

E-mail:valdirene@cse.ufsc.br

Rogério João Lunkes

Pós-Doutorado pela Universidade de Valencia

Professor Associado da Universidade Federal de Santa Catarina - UFSC Reitor João David Ferreira Lima. Bairro Trindade. Florianópolis/SC. CEP 88040-900

E-mail: lunkes@cse.ufsc.br

Darci Schnorrenberger

Doutorado em Engenharia de Produção pela Universidade Federal de Santa

Catarina - UFSC

Professor Adjunto da Universidade Federal de Santa Catarina - UFSC Reitor João David Ferreira Lima. Bairro Trindade. Florianópolis/SC. CEP 88040-900

E-mail: darcisc@gmail.com

\section{RESUMO}

O acirramento da competição faz com que as empresas preocupem-se com a racionalização dos recursos aplicados na produção, busquem melhorar seus processos produtivos e a gestão das informações, visando aumentar a competitividade e expandir o seu mercado. As informações de custos podem auxiliar nesta tarefa, razão pela qual este trabalho discute a aplicação do método da unidade de esforço de produção (UEP) em uma empresa fabricante de cosméticos. Foi realizado um estudo de caso em uma empresa do Estado de Santa Catarina, na estruturação do método da UEP, envolvendo as cinco etapas da fase de implantação, que são concluídas com a determinação dos equivalentes dos produtos em UEP. A partir destes equivalentes faz-se a operacionalização do método para a produção do período subsequente ao da implantação, chegando-se ao custo de transformação de cada produto. Com o método, a empresa pode apurar mensalmente, de forma facilitada, o custo de transformação unitário dos produtos que fabrica, tendo assim informações que auxiliam no controle e tomada de 
decisões. O método da UEP facilita o processo de apuração de custos, sendo essa uma de suas grandes vantagens em relação aos demais métodos de custeio.

Palavras-chave: Custos. Unidade de esforço de produção. Cosméticos.

\title{
The application of the Unit of Production Effort Method (UEP): study in a cosmetics company
}

\begin{abstract}
The present market leads the companies to be concerned with the rationalization of the resources applied on the production, to seek to improve their production processes and information management, to increase the competitiveness and to expand the market. The cost information can aid in this task, reason why this work discuss the application of the Unit of Production Effort Method (UEP) in a cosmetics manufacturing company. A study was carried in a company of State of Santa Catarina, in the structuring of the method, involving the five stages of the implantation phase, that are concluded with the equivalent of the products in UEP determination. From these equivalent ones it is made the operationalization of the method for the production to the period following the implantation, obtaining the transformation cost of each product. On the basis of that method, the company may calculating easily, every month, the unitary transformation cost of the products that manufactures, supporting the control and decision making. The UEP method facilitates the costing process. This is one of the benefits when compared to other costing methods.
\end{abstract}

Key-words: Costs. Unit of Production Effort Method. Cosmetics.

\section{INTRODUÇÃO}

O conhecimento dos custos envolvidos no processo de produção de um bem auxilia as empresas no controle e tomada de decisão (BORNIA, 2009). Borgert e Scharf (2008) completam, afirmando que a preparação das informações de custos deve estar focada em fornecer subsídios suficientes para que, analisados em conjunto com as demais variáveis, fundamentem a tomada de decisão.

Entre os mercados de grande crescimento no Brasil, está o de cosméticos. Isto inclui a vinda de empresas multinacionais, que estão buscando esse mercado, que é o terceiro maior do mundo (ABIHPEC, 2012; DAVID, 2012). Além disso, as empresas são diariamente pressionadas e questionadas por um consumidor mais informado e exigente, que busca muito mais do que preço baixo (DAVID, 2012). 
Para uma organização manter-se competitiva neste segmento é fundamental conhecer os custos dos diferentes produtos fabricados.

Assim, uma metodologia de custos que disponibilize informações confiáveis é um instrumento gerencial relevante, e cada empresa precisa de um modelo desenhado especificamente para suas necessidades e características, já que, conforme lembra Maher (2001), em custos necessidades diferentes exigem informações diferentes, e por isso a ideia de tamanho único não se aplica na área de custos.

Dentre os métodos de custeio desenvolvidos há o da unidade de esforço de produção (UEP), que busca a criação de uma unidade comum de medida capaz de quantificar produtos de diferentes naturezas como se fossem iguais, simplificando o processo de apuração de custos e de gestão da produção (SILVA, 2006). Este método é empregado nesse estudo, que tem o objetivo de discutir sua utilização a partir do estudo em uma empresa fabricante de cosméticos.

Para atender a esse objetivo, o o estudo está organizado em cinco seções, iniciando-se por esta introdução. Na segunda seção é apresentado o referencial teórico que sustenta a pesquisa, na terceira se discute os procedimentos metodológicos, e após apresentam-se os dados e discussões da pesquisa. A quinta seção mostra as conclusões, seguidas pelas referências.

\section{REFERENCIAL TEÓRICO}

Métodos de custeio referem-se às formas como as informações são obtidas, ou seja, como os dados são tratados para a obtenção das informações, envolvendo o processo operacional para obtenção dessas informações de custos (BORNIA, 2009).

$\mathrm{Na}$ definição da melhor opção para gestão de custos, dependendo das características da organização, do ramo de atividade, dos produtos, estrutura de gastos, disponibilidade de recursos para manutenção do método, e dos objetivos em relação às informações de custos, pode ser empregado um ou outro método de custeio, como: 
- Custeio por absorção, que pode ser sem ou com departamentalização, também denominado de método dos centros de custos;

- Custeio pleno ou BAB (Betriebsabrechnungsbogen), que corresponde a um mapa de distribuição dos custos desenvolvido pelo RKW (Reichskuratorium für Wirtschaftlichtkeit), centro de estudos conduzido pelo governo alemão (DE $\mathrm{ROCCHI}, 2007$ );

- Custeio variável, em que somente os custos variáveis são alocados (usado para custeio de objetos e análise de lucratividade/margem);

- Custeio direto, em que se alocam somente os custos diretos (usado para avaliação de desempenho);

- $\mathrm{ABC}$ (Custeio Baseado em Atividades) ou TDABC (time driven activity-based costing); e

- UEP (Unidade de Esforço de Produção).

Na sequência apresenta-se uma discussão sobre o método da unidade de esforço de produção (UEP), escolhido para o desenvolvimento desse estudo.

\subsection{Origem e Características do Método da UEP}

Na década de 1940, o francês Georges Perrin desenvolveu um método de cálculo e alocação de custos e controle de gestão, que denominou de Georges Perrin Method (GPM) (ALLORA; GANTZEL, 1996; BORNIA, 2009; LA VILLARMOIS; LEVANT, 2011). Perrin tentou proteger sua invenção, o que fez com que o GPM também fosse pouco disseminado, chegando a um número entre 150 e 200 implementações (ALCOUFFE; BERLAND; LEVANT, 2008). A partir de 1977 foi reiniciada a utilização do método na França, por uma empresa de consultoria que o renomeou de Método UP, sendo depois de 1995 novamente renomeado para Método UVA (Added Value Unit Method) (ALCOUFFE, BERLAND e LEVANT, 2008; LA VILLARMOIS; LEVANT, 2011).

No Brasil, o desenvolvimento da obra de Perrin contou com a colaboração do engenheiro Franz Allora, seu discípulo, que divulgou o instrumento como Método da UP (Unidade de Produção) ou UEP (Unidade de Esforço de Produção). Allora veio para o Brasil no início dos anos sessenta e criou em 1978 uma empresa de 
consultoria em Blumenau, no estado de Santa Catarina, cuja atividade principal era a aplicação do método (BORNIA, 2009; ALLORA; GANTZEL, 1996).

O método da UEP, como é mais conhecido no meio acadêmico (BORNIA, 2009), "consiste na construção e utilização de uma unidade de medida dos esforços e recursos aplicados na produção de vários produtos. Essa medida deve ser homogênea, de forma que possa servir de denominador comum a todos os produtos" (MARTINS, 2008, p. 312).

São tratados no método os custos de transformação, que compreendem custos de mão-de-obra direta e custos indiretos de fabricação, porém, pode ser empregado em conjunto com outros métodos para alocação das despesas, como $A B C$ e RKW.

Este instrumento de custeio possibilita a soma de grandezas não homogêneas em uma indústria multiprodutora, por exemplo, visando simplificar a obtenção das informações de custos e deixá-las similares às das indústrias que produzem somente um produto, unificando a produção. Para isso, determina uma unidade de medida comum a todos os produtos da empresa, a UEP (KLIEMANN NETO, 1995; BORNIA, 2009; SILVA, 2006).

No método da UEP a unificação da produção está relacionada ao conceito teórico de esforço de produção, que representa os esforços despendidos no processo de transformação da matéria-prima em produto acabado (BORNIA, 2009; KLIEMANN NETO, 1995). A noção de esforço de produção possui como característica a homogeneidade, que permite afirmar que quaisquer que sejam os artigos produzidos e seus respectivos processos, estes necessitam de esforços de produção de mesma natureza, embora de intensidades diferentes (KLIEMANN NETO, 1995).

Os esforços de produção são concentrados nas atividades diretamente envolvidas na fabricação dos produtos, assim, os esforços das atividades auxiliares são repassados às produtivas, e daí aos produtos (BORNIA, 2009).

Na sequência apresenta-se a sistemática de alocação de custos no método da UEP. O método é dividido em duas fases: a primeira é a fase de implantação, que culmina com a determinação dos equivalentes em UEP de cada produto. Essa fase é a mais demorada, porém, ocorre quando o método é implantado e após, 
Utilização do Método da Unidade de Esforço de Produção (UEP): estudo em uma empresa de cosméticos.

Guilherme Luiz, Valdirene Gasparetto, Rogério João Lunkes, Darci Schonrrenberger

novamente, apenas quando há mudanças substanciais em produtos ou no processo produtivo. A segunda fase é a operacionalização, que ocorre em todos os períodos, porém, é mais fácil e rápida de executar, demandando poucos recursos e tempo, sendo essa característica uma das grandes vantagens do método (BORNIA, 2009; LA VILLARMOIS; LEVANT, 2011).

\subsection{Implantação do Método da UEP}

A implantação pode ser dividida em cinco etapas, ilustradas na Figura 1 e detalhadas na sequência.

\section{- Divisão da Fábrica em Postos Operativos (PO)}

Um PO pode ser definido como uma ou mais operações simples e homogêneas, ou seja, da mesma natureza para todos os produtos que passarem por ele, mudando somente o tempo de passagem dos produtos (KLIEMANN NETO, 1995).

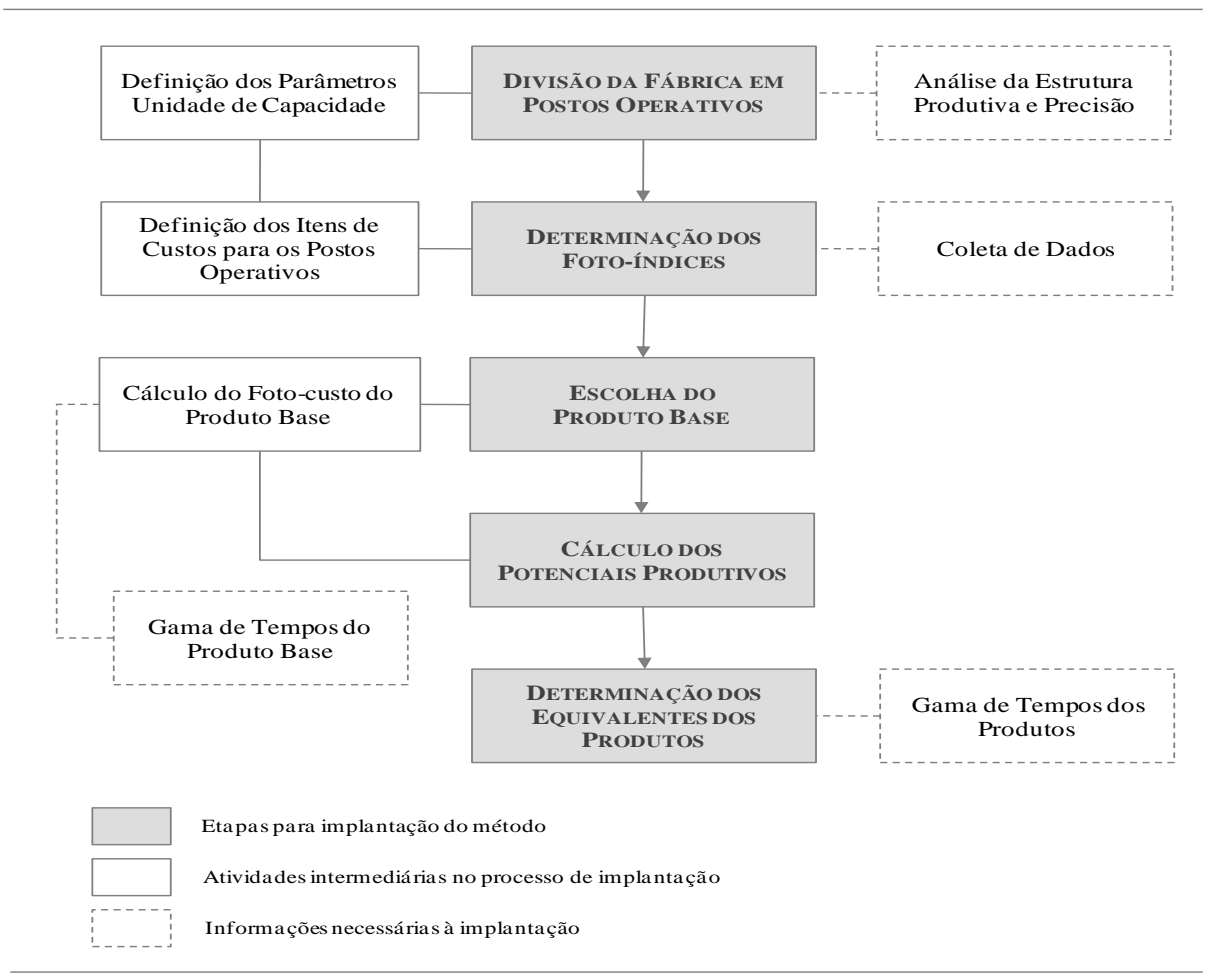

Figura 1 - Roteiro para a implantação do método da UEP.

Fonte: Adaptado de Kliemann Neto (1995) 
Maior precisão na unidade de esforço de produção é obtida utilizando-se maior homogeneidade dos POs (IAROZINSKI NETO, 1989), sendo o grau de exatidão da informação também relacionado à quantidade de POs utilizados na implantação do método. Entretanto, deve-se avaliar a relação custo-benefício, pois quanto mais POs, mais onerosa será a implantação (SILVA, 2006).

Conforme Silva (2006), uma máquina ou um conjunto de máquinas que realizam a mesma função, um operário ou um conjunto destes, podem ser considerados POs. O mapeamento do processo de produção pode auxiliar na determinação destes postos.

\section{- Determinação dos Foto-índices dos POs}

Os foto-índices representam o custo horário dos POs e "são calculados tecnicamente, de acordo com o efetivo dispêndio de insumos por parte dos postos operativos em funcionamento, com exceção de matérias-primas e despesas de estrutura" (BORNIA, 2009, p. 144). Silva (2006) complementa, afirmando que devem ser incluídos no cálculo os custos de transformação passíveis de serem relacionados ao PO, divididos pelo total de horas trabalhadas no mês.

\section{- Escolha do Produto Base}

O produto base deve ser um produto real ou fictício que apresente de forma mais representativa a estrutura da empresa. A opção deve ser pelo produto que passe pelo maior número de $\mathrm{POs}$ possível, ou pelos mais significativos do processo (ANTUNES JR., 1988).

O produto base vale uma UEP, e o número de UEPs dos demais é calculado em função deste (SILVA, 2006).

\section{- Cálculo dos Potenciais Produtivos}

Potencial produtivo é a capacidade de gerar esforço de produção, em UEP/h, ou seja, é a quantidade de esforços gerados pelo PO por hora de funcionamento (BORNIA, 2009). 


\section{- Determinação dos Equivalentes dos Produtos}

Equivalente dos produtos "é a somatória de todos os esforços de produção absorvidos pelos produtos na medida em que passam pelos postos operativos" (SILVA, 2006, p. 44), consistindo no valor, em UEP, de cada produto (BERNARDES, 1999). Refere-se à soma dos resultados obtidos a partir da multiplicação entre o tempo de passagem de cada um dos produtos nos POs e o valor do potencial produtivo dos respectivos postos (SILVA, 2006).

Após o cálculo dos equivalentes dos produtos, está finalizada a fase de implantação deste método.

\subsection{Operacionalização do Método da UEP}

A segunda fase da aplicação do método da UEP é a operacionalização, que segundo Medeiros (2009) e Antunes Jr. (1988), objetiva valorar monetariamente a UEP.

Mensura-se a produção total do período em UEPs, somando-se os resultados obtidos a partir da multiplicação dos equivalentes em UEP dos produtos, pela quantidade física destes (MEDEIROS, 2009). Após divide-se os custos de transformação incorridos no período pela produção total da fábrica em UEP, obtendo-se o valor monetário de uma UEP no período. Calculam-se os custos de transformação dos produtos multiplicando-se este valor pela quantidade de UEPs de cada produto (BERNARDES, 1999).

Para executar os procedimentos necessários ao método, empregando uma planilha de cálculo, é suficiente a metade do tempo de trabalho de um colaborador (LA VILLARMOIS; LEVANT, 2011), devido à simplicidade dos procedimentos envolvidos.

O cálculo do custo de transformação dos produtos completa a segunda fase e conclui a aplicação do método. 


\section{PROCEDIMENTOS METODOLÓGICOS}

Esta pesquisa caracteriza-se como descritiva quanto ao seu objetivo, por registrar, analisar e correlacionar fatos sem manipulá-los, além de procurar descobrir a frequência de ocorrência de um fenômeno (CERVO; BERVIAN; SILVA, 2007).

Quanto à abordagem do problema este estudo é predominantemente qualitativo, sem desconsiderar a abordagem quantitativa em etapas relacionadas à implantação do método da UEP, como a distribuição de custos comuns aos postos operativos a partir de critérios de alocação. Minayo (2000) lembra que uma pesquisa pode ser classificada como qualitativa e quantitativa, já que estes dois tipos de pesquisa não se opõem, ao contrário, completam-se, pois, a realidade abrangida por elas interage dinamicamente, excluindo qualquer dicotomia.

Quanto aos procedimentos para coleta dos dados, que para Raupp e Beuren (2003, p.83) "referem-se à maneira pela qual se conduz o estudo e, portanto, se obtêm os dados", este estudo configura-se como bibliográfico e de caso único, realizado em uma empresa brasileira fabricante de cosméticos, fundada em 1990, em Santa Catarina.

$\mathrm{Na}$ implantação do método da UEP, consideraram-se os produtos usuais de fabricação da empresa, sendo analisados 197 itens. Cada produto foi considerado na forma como é comercializado no atacado pela empresa fabricante. Assim, uma unidade de produto representa a embalagem final, que é composta por 6 ou 12 unidades de embalagens primárias, com exceção da linha galão, cujos produtos não possuem embalagem final, sendo comercializados na embalagem primária - assim, uma unidade de produto desta linha representa uma embalagem primária.

Os dados foram coletados durante três meses, sendo que os custos e as horas de funcionamento referem-se à produção ocorrida em um mês de operação da empresa. As horas de funcionamento de alguns postos operativos foram extraídas das ordens de produção ou informadas pela supervisão da produção ou por empregados da fábrica. Assim, os dados foram coletados a partir de visitas à empresa e também junto aos empregados da área produtiva e financeira. 
$\mathrm{Na}$ discussão da operacionalização do método, foram utilizados dados de produção do mês seguinte ao de implantação. Porém, foi considerado um valor fictício de custos de transformação, apenas para ilustração desta etapa do método.

Um fator limitante deste método e do próprio estudo refere-se ao fato de o método trabalhar com os custos de transformação, o que implica o não tratamento dos custos de matéria-prima e outros materiais diretos, como embalagem, e das despesas de estrutura (BORNIA, 2009).

$\mathrm{Na}$ cronometragem dos tempos de passagem dos produtos nos postos operativos, para cálculo do foto-custo base e dos equivalentes dos produtos, não foram consideradas a ociosidade e setups de máquina, pela dificuldade de mensuração.

Por fim, apesar do roteiro geral de concepção e implantação ser padronizado, o resultado final desta pesquisa limita-se à estruturação do método da UEP em uma empresa fabricante de cosméticos, que possibilita a apuração dos custos de transformação dos produtos elaborados por esta empresa apenas, não sendo os resultados passíveis de generalização, mesmo que para empresas do mesmo ramo de atividade e com características similares.

\section{APRESENTAÇÃO E ANÁLISE DOS DADOS}

Esta seção apresenta a empresa onde o estudo foi realizado, seu processo produtivo e descreve a estruturação do método da UEP, detalhando os procedimentos executados nas fases de implantação e operacionalização.

\subsection{A Empresa}

A empresa pesquisada desenvolve produtos para tratamento capilar, corporal, facial e de higiene, e dispõe de uma equipe comercial interna, distribuidores e consultores atuando no país e dezessete lojas franqueadas para distribuí-los. Atualmente ela gera 50 empregos diretos. Seu organograma é apresentado na Figura 2. 


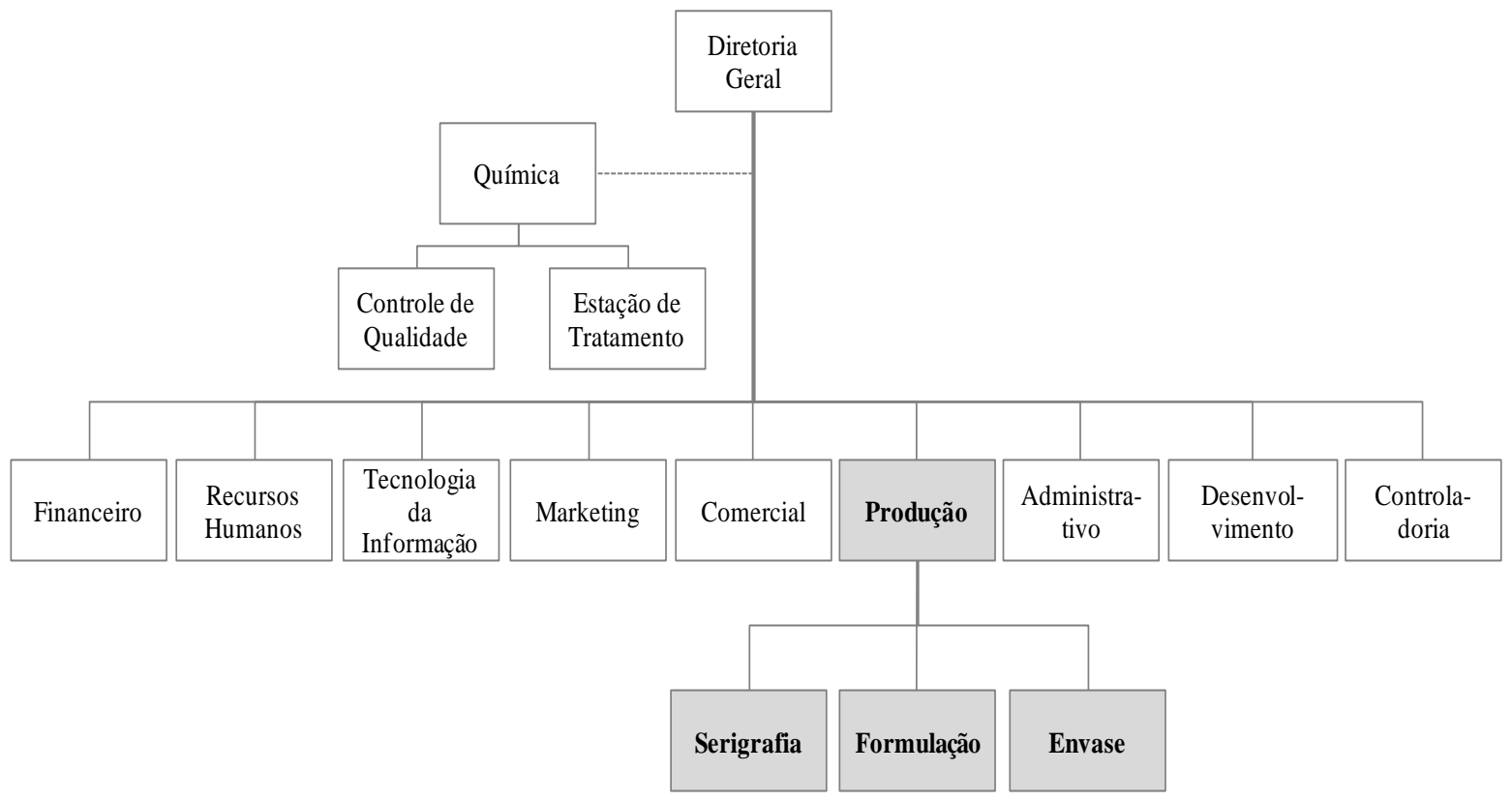

Figura 2 - Organograma da empresa pesquisada Fonte: Elaborado pelos autores.

O Departamento de Produção, onde se concentram os custos de transformação, está destacado na Figura 2, compreendendo Serigrafia, Formulação e Envase, onde o fluxo do processo produtivo está concentrado, conforme Figura 3.

A Serigrafia é responsável pela impressão das informações que devem constar nas embalagens dos produtos, já que parte das embalagens é comprada neutra e estas informações são estampadas na fábrica. Na Formulação ocorre a elaboração dos cosméticos, enquanto o Envase é responsável por inserir os cosméticos nas embalagens e prepará-los para a comercialização. A partir desta etapa, o produto está pronto para ser expedido ou estocado.

\subsection{Utilização do Método da Unidade de Esforço de Produção}

O método da UEP foi aplicado em conformidade com o que foi tratado no referencial teórico.

\subsubsection{Implantação do Método da UEP}

A implantação envolveu cinco etapas descritas a seguir. 
Utilização do Método da Unidade de Esforço de Produção (UEP): estudo em uma empresa de cosméticos. Guilherme Luiz, Valdirene Gasparetto, Rogério João Lunkes, Darci Schonrrenberger

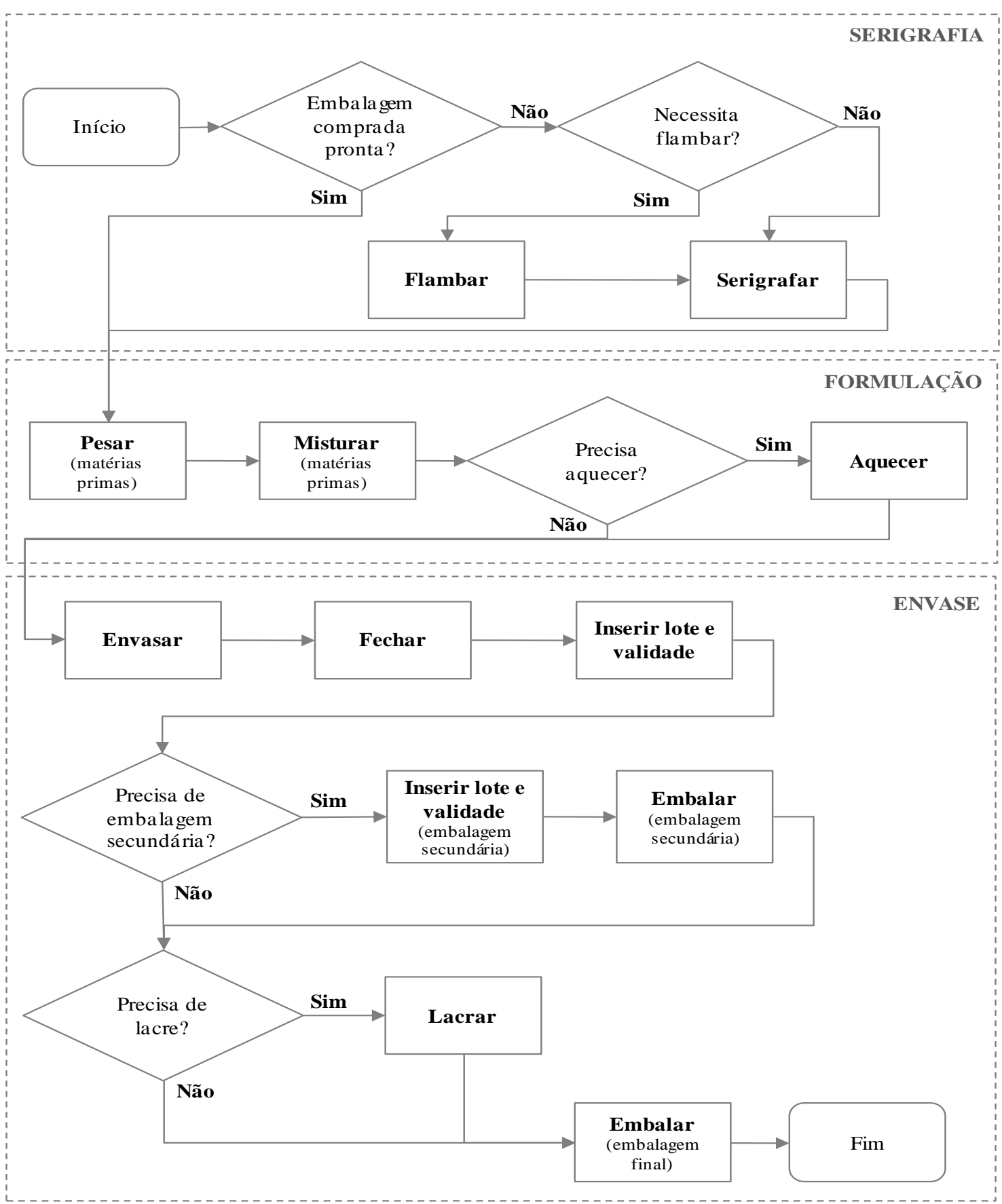

Figura 3 - Fluxo do processo produtivo da empresa pesquisada Fonte: Elaborado pelos autores.

- Divisão da fábrica em postos operativos

Foram definidos 17 POs na empresa, conforme Quadro 1. Alguns POs possuem denominações semelhantes, mas há características que os distinguem. Por exemplo, o Envase de Líquidos 1 é representado por uma máquina que 
Utilização do Método da Unidade de Esforço de Produção (UEP): estudo em uma empresa de cosméticos. Guilherme Luiz, Valdirene Gasparetto, Rogério João Lunkes, Darci Schonrrenberger

preenche embalagens de 100 e 500 ml e o Envase de Líquidos 2 destina-se às demais embalagens.

\begin{tabular}{|c|c|c|c|}
\hline PO & Nome do PO & PO & Nome do PO \\
\hline 01 & Flambagem & 10 & Envase Líquidos 1 \\
\hline 02 & Serigrafia 1 & 11 & Envase Líquidos 2 \\
\hline 03 & Serigrafia 2 & 12 & Envase Semi-sólidos \\
\hline 04 & Pesagem & 13 & Fechamento \\
\hline 05 & Mistura de Líquidos e Semi-sólidos & 14 & Impressão \\
\hline 06 & Mistura Manual & 15 & Lacre \\
\hline 07 & Mistura de Sólidos & 16 & Embalagem Secundária \\
\hline 08 & Aquecimento & 17 & Embalagem Final \\
\hline 09 & Envase Manual & & \\
\hline
\end{tabular}

Quadro 1 - Determinação dos POs

\section{- Determinação dos foto-índices}

Essa etapa refere-se à determinação do custo horário $(R \$ / h)$ dos $P O s$, apresentada no Quadro 2 (para parte dos POs). No processo de alocação dos custos aos POs é utilizado um critério para cada item de custo, a partir da análise do seu fator causal, como também faz-se nos demais métodos de custeamento.

\begin{tabular}{|c|c|c|c|}
\hline POs & $\begin{array}{c}\text { Total - custos de } \\
\text { Transformação }\end{array}$ & $\begin{array}{c}\text { Horas } \\
\text { funcionamento }\end{array}$ & $\begin{array}{c}\text { Foto-índices } \\
(\mathbf{R} \mathbf{\text { h }})\end{array}$ \\
\hline 01 & $1.148,69$ & 42 & $\mathbf{2 7 , 3 5}$ \\
\hline 02 & $5.380,46$ & 83 & $\mathbf{6 4 , 8 2}$ \\
\hline 03 & $4.367,30$ & 150 & $\mathbf{2 9 , 1 2}$ \\
\hline$\ldots$ & $\ldots$ & $\ldots$ & $\ldots$ \\
\hline 17 & $2.802,11$ & 140 & $\mathbf{2 0 , 0 2}$ \\
\hline Total & $\mathbf{4 1 . 5 1 7 , 3 6}$ & & \\
\hline
\end{tabular}

Quadro 2 - Foto-índices dos POs em custo/hora

\section{- Escolha do produto base}

O produto 179 é líder de vendas na empresa, e foi selecionado como produto base por sugestão dos gestores e por passar pelos POs mais representativos da fábrica. 
Utilização do Método da Unidade de Esforço de Produção (UEP): estudo em uma empresa de cosméticos. Guilherme Luiz, Valdirene Gasparetto, Rogério João Lunkes, Darci Schonrrenberger

Para cálculo do foto-custo base multiplicam-se os foto-índices dos POs pelo tempo que o produto base demanda em sua passagem nestes POs, conforme Quadro 3.

\begin{tabular}{|c|c|c|c|}
\hline POs & $\begin{array}{l}\text { Foto-índices dos } \\
\text { POs }(\$ / h)\end{array}$ & $\begin{array}{c}\text { Tempo de } \\
\text { passagem do } \\
\text { produto base }(\mathrm{h})\end{array}$ & $\begin{array}{l}\text { Foto-custo } \\
\text { base (\$) }\end{array}$ \\
\hline 01 & 27,35 & 0,01167 & 0,32 \\
\hline 02 & 64,82 & 0,00000 & - \\
\hline 03 & 29,12 & 0,00917 & 0,27 \\
\hline 04 & 48,56 & 0,00400 & 0,19 \\
\hline 05 & 10,14 & 0,03600 & 0,37 \\
\hline 06 & 100,25 & 0,00000 & - \\
\hline 07 & 79,44 & 0,00000 & - \\
\hline 08 & 12,89 & 0,01800 & 0,23 \\
\hline 09 & 183,85 & 0,00000 & - \\
\hline 10 & 101,05 & 0,00000 & - \\
\hline 11 & 224,08 & 0,00000 & - \\
\hline 12 & 35,65 & 0,01050 & 0,37 \\
\hline 13 & 20,72 & 0,00517 & 0,11 \\
\hline 14 & 17,8 & 0,00811 & 0,14 \\
\hline 15 & 24,26 & 0,01286 & 0,31 \\
\hline 16 & 25,98 & 0,00000 & - \\
\hline 17 & 20,02 & 0,01750 & 0,35 \\
\hline \multicolumn{3}{|c|}{ Valor de 1 UEP } & 2,67 \\
\hline
\end{tabular}

Quadro 3 - Foto-custo base

\section{- Cálculo dos Potenciais Produtivos}

Os foto-índices dos POs são divididos pelo foto-custo base, conforme Quadro 4, e representam a capacidade dos POs de gerar esforço de produção por hora de funcionamento (UEP/h).

\begin{tabular}{|c|c|c|c|}
\hline $\begin{array}{l}\text { Postos } \\
\text { operativos }\end{array}$ & $\begin{array}{c}\text { Foto-índice dos } \\
\text { postos operativos } \\
(\mathrm{R} \$ / \mathrm{h})\end{array}$ & $\begin{array}{l}\text { Foto-custo } \\
\text { base }(R \$)\end{array}$ & $\begin{array}{c}\text { Potenciais } \\
\text { Produtivos (UEP/h) }\end{array}$ \\
\hline 01 & 27,35 & 2,67 & 10,2 \\
\hline 02 & 64,82 & 2,67 & 24,33 \\
\hline 03 & 29,12 & 2,67 & 10,92 \\
\hline$\ldots$ & $\ldots$ & $\ldots$ & $\ldots$ \\
\hline 17 & 20,02 & 2,67 & 7,52 \\
\hline
\end{tabular}

Quadro 4 - Potenciais produtivos dos POs 
Utilização do Método da Unidade de Esforço de Produção (UEP): estudo em uma empresa de cosméticos. Guilherme Luiz, Valdirene Gasparetto, Rogério João Lunkes, Darci Schonrrenberger

\section{- Determinação dos Equivalentes dos Produtos}

Para determinação dos equivalentes em UEP dos produtos no momento de implantação do método, multiplica-se o tempo de passagem dos produtos nos POs pelo seu potencial produtivo, conforme Quadro 5.

\begin{tabular}{|c|c|}
\hline Código do Produto & UEP \\
\hline 001 & 1,23 \\
\hline 002 & 1,12 \\
\hline 003 & 2,52 \\
\hline 004 & 1,13 \\
\hline 005 & 1,43 \\
\hline$\ldots$ & $\ldots$ \\
\hline 111 & 6,60 \\
\hline$\ldots$ & $\ldots$ \\
\hline $\mathbf{1 7 9}$ & $\mathbf{1 , 0 0}$ \\
\hline$\ldots$ & $\ldots$ \\
\hline 197 & 1,61 \\
\hline
\end{tabular}

Quadro 5 - Valores em UEP dos produtos

O produto de código 179 é o produto base, por isso seu valor em UEP é 1,00. O equivalente do produto 111 é 6,60 UEP. Este valor elevado é determinado principalmente pelo tempo de passagem do produto pelos POs 06 e 09, que são mais onerosos.

\subsubsection{Operacionalização do Método da UEP}

O Quadro 6 mostra a mensuração da produção física da empresa ocorrida no mês seguinte ao de implantação. Com base nos equivalentes dos produtos, apurase a produção em UEP da fábrica. Essa etapa deve ser realizada todos os meses.

Como se observa no Quadro 6, a produção em UEPs da fábrica nesse mês resultou no montante de 3.765,99 UEPs.

\begin{tabular}{|c|c|c|c|}
\hline $\begin{array}{c}\text { Código do } \\
\text { produto }\end{array}$ & $\begin{array}{c}\text { Quantidade } \\
\text { produzida }\end{array}$ & $\begin{array}{c}\text { Equivalentes dos } \\
\text { produtos em UEP }\end{array}$ & $\begin{array}{c}\text { Quantidade } \\
\text { produzida em UEP }\end{array}$ \\
\hline 005 & 40 & 1,43 & 57,20 \\
\hline 010 & 40 & 1,21 & 48,40 \\
\hline 026 & 39 & 2,00 & 78,00 \\
\hline$\ldots$ & $\ldots$ & $\ldots$ & $\ldots$ \\
\hline Total & $\mathbf{1 . 8 4 4}$ & & $\mathbf{3 . 7 6 5 , 9 9}$ \\
\hline
\end{tabular}

Quadro 6 - Produção da empresa em UEP no mês seguinte ao de implantação 
Supondo-se que os custos de transformação da empresa representaram $\mathrm{R} \$$ $40.000,00$, o valor monetário de uma UEP corresponde a: $R \$ 40.000,00$ / 3.765,99 $\mathrm{UEPs}=\mathrm{R} \$ 10,62$.

O Quadro 7 apresenta o cálculo dos custos de transformação dos produtos apresentados no Quadro 6.

Quanto aos custos utilizados no cálculo, os gestores da empresa devem avaliar as informações que desejam obter. Pode se considerar somente os custos de transformação, mas pode se também alocar as despesas de estrutura, como fez Bernardes (1999) em um estudo de caso, ou pode ser empregado outro método de custeio para tratar as despesas como propõem Allora e Gantzel (1996). Para apurar o custo total dos produtos é preciso incluir ainda os custos de matéria prima e outros materiais diretos, como embalagem.

\begin{tabular}{|c|c|c|c|}
\hline $\begin{array}{c}\text { Código do } \\
\text { produto }\end{array}$ & $\begin{array}{c}\text { Equivalentes dos } \\
\text { produtos em } \\
\text { UEP }\end{array}$ & $\begin{array}{c}\text { Valor monetário da } \\
\text { UEP (R\$) }\end{array}$ & $\begin{array}{c}\text { Custo de transformação } \\
\text { com base no método da } \\
\text { UEP (R\$) }\end{array}$ \\
\hline 005 & 1,43 & 10,62 & 15,19 \\
\hline 010 & 1,21 & 10,62 & 12,85 \\
\hline 026 & 2,00 & 10,62 & 21,24 \\
\hline
\end{tabular}

Quadro 7 - Custo de transformação dos produtos baseado no método da UEP

Antes da realização deste estudo não se apurava custos por produtos na empresa. Elaborava-se, para fins gerenciais, uma demonstração do resultado mensal (DRE) da empresa como um todo, que era utilizada para avaliação do desempenho financeiro.

Com a utilização do método da UEP, os gestores da empresa têm mais informações para o planejamento e determinação de estratégias em relação a preços e mix de produção, já que as informações sobre os equivalentes dos produtos mostram as relações entre os produtos em termos de utilização dos esforços de produção. Além disso, o método possibilita, de forma simples, o cálculo do custo de produção dos itens fabricados. 
Essas informações permitirão maior segurança na tomada de decisões relacionadas aos produtos o que pode auxiliar na melhoria do seu desempenho e competitividade.

\section{CONSIDERAÇÕES FINAIS}

Para as empresas que atuam em mercados competitivos, racionalizar custos, melhorar os processos produtivos e tomar decisões acertadas, são ações que contribuem para a sobrevivência. Nesse sentido, as informações de custos são relevantes, pois conjugadas com outras, embasam as ações mencionadas.

A proposta desse estudo foi de discutir as etapas de utilização do método da UEP em uma organização fabricante de cosméticos. O método possui duas fases distintas: implantação e operacionalização. A fase de implantação é a mais complexa e está dividida em cinco etapas, e seu resultado final é o equivalente em UEP dos produtos.

Já a fase de operacionalização é facilitada pelo método, uma vez que se apura o montante de produção física, a produção em UEP e em seguida, apropria-se os custos de transformação a essa produção. Esta etapa demanda pouco tempo e recursos.

$\mathrm{O}$ método da UEP mostrou-se adequado à empresa estudada, que possui processos produtivos padronizados, permitindo a definição dos postos operativos e a manutenção das relações entre os equivalentes em UEP dos produtos por um período relativamente longo.

Constata-se, assim, que o método da UEP é uma alternativa para apuração de custos em empresas multiprodutoras, gerando informações confiáveis, contribuindo no apoio à tomada de decisões mais embasadas e em tempo hábil, resultando na melhoria do desempenho e aumento da competitividade da organização.

Resta, no entanto, tornar a alertar que, apesar do roteiro de concepção e implantação ser padronizado, os resultados decorrentes de sua aplicação possuem validade restrita à organização para a qual foi estruturado o método, devendo 
sempre ser implantado à luz das características da organização que utilizará o método da UEP.

\section{REFERÊNCIAS}

ABIHPEC - Associação Brasileira da Indústria de Higiene pessoal, Perfumaria e Cosméticos). (2012). São Paulo. Disponível em: http://www.abihpec.org.br.

ALCOUFFE, Simon; BERLAND, Nicolas; LEVANT, Yves. (2008). Actor-networks and the diffusion of management accounting innovations: a comparative study. Management Accounting Research, v. 19, p. 1-17.

ALLORA, Valerio; GANTZEL, Gerson. (1996). Revolução nos custos: os metodos $A B C$ e UP e a gestão estratégica de custos como ferramenta para a competitividade. Salvador: Casa da Qualidade.

ANTUNES JUNIOR, Jose Antonio Valle. (1988). Fundamentação do método das unidades de esforço de produção. (Dissertação de Mestrado). Universidade Federal de Santa Catarina, Florianópolis.

BERNARDES, Simone. (1999). Implementação do método da unidade de esforço de produção em uma pequena indústria moveleira em Santa Catarina: estudo de caso. (Dissertação de Mestrado). Universidade Federal de Santa Catarina, Florianópolis.

BORGERT, Altair; SCHARF, Luciano. (2008). Custo exato: possibilidade e necessidade. SIMPÓSIO DE ADMINISTRAÇÃO DA PRODUÇÃO LOGÍSTICA E OPERAÇÕES INTERNACIONAIS, 11, São Paulo, Anais. Fundação Getúlio Vargas, 2008.

BORNIA, Antonio Cezar. (2009). Análise gerencial de custos: aplicação em empresas modernas. (2 ed.). São Paulo: Atlas.

BORNIA, Antonio Cezar. (2009). Análise gerencial de custos: aplicação em empresas modernas. (2 ed.). São Paulo: Atlas.

CERVO, A. L.; BERVIAN, P. A.; SILVA, R. da. (2007). Metodologia cientifica. (6 ed.). São Paulo: Pearson Prentice Hall.

DAVID, R. (2012). A Competitividade no Mercado de Cosméticos. Disponível em: http://www.guiasalaobrasil.com/coluna_rogerio_david_4.php

DE ROCCHI, Carlos Antonio. (2007). Transferência internacional de tecnologia contábil: um estudo comparativo numa perspectiva geográfica-temporal. (Tese de Doutorado). Universidade Federal de Santa Catarina, Florianópolis. 
IAROZINSKI NETO, Alfredo. (1989). A gestão industrial através do método das unidades de esforço de produção (UEP's). (Dissertação de Mestrado). Universidade Federal de Santa Catarina, Florianópolis.

KLIEMANN NETO, Francisco José. (1995). Gerenciamento e controle da produção pelo método das unidades de esforço de produção. CONGRESSO BRASILEIRO DE GESTÃO ESTRATÉGICA DE CUSTOS, 1, 1995, São Leopoldo, Anais. Unisinos.

LA VILLARMOIS. Olivier de. LEVANT, Yves. (2011). From adoption to use of a management control tool: case study evidence of a costing method. Journal of Applied Accounting Research, v. 12, n. 3, p. $234-259$.

MAHER, Michael. (2001). Contabilidade de custos: criando valor para a administração. São Paulo: Atlas.

MARTINS, Eliseu. (2008). Contabilidade de custos. (9 ed.). São Paulo: Atlas.

MEDEIROS, Renato Felipe. (2009). Adaptação do método das unidades de esforço de produção (UEP), para empresas de cerâmicas vermelha. (Dissertação de Mestrado). Universidade Federal de Santa Catarina, Florianópolis.

MINAYO, Maria Cecília de Souza. (2000). O desafio da pesquisa social. In: MINAYO, Maria Cecília de Souza (Org). Pesquisa social: teoria, método e criatividade. Petrópolis: Vozes.

RAUPP, Maury Fabiano; BEUREN, Ilse Maria. Metodologia da pesquisa aplicável às ciências sociais. In: BEUREN, Ilse Maria (Org). (2003). Como elaborar trabalhos monográficos em contabilidade: teoria e prática. São Paulo: Atlas.

SILVA, Márcia Zanievicz da. (2006). Mensuração dos custos de procedimentos médicos em organizações hospitalares: sistematização de um método de custeio híbrido à luz do ABC e da UEP. (Dissertação de Mestrado). Universidade Federal de Santa Catarina.

Data de Submissão: 19/02/2013

Data de Aceite: 26/05/2014 